\title{
Methods used to maintain a high level of participant involvement in a clinical trial
}

\author{
M E Hellard, M I Sinclair, A B Forbes, C K Fairley
}

\begin{abstract}
Objectives-To describe the strategies adopted to maintain high level participation throughout a community based clinical trial, and the reasons given by participants for why they participated in the study.
\end{abstract}

Design-Observational study.

Setting-Community based clinical trial in Melbourne, Australia that ran for 68 weeks and involved 2811 community based individuals from 600 families. A high level of commitment was required of the families; each participant completed a Health Diary each week of the 68 week study, as well as answering numerous other questionnaires, and providing faecal and blood samples.

Main results-Only 41 of the 600 families withdrew from the study; the majority of these families withdrew because they sold their home and moved from the study area. The completion rate of Health Diaries averaged $90.7 \%$ over the 68 weeks of data collection. Of the $\mathbf{5 5 9}$ families who completed the study, $524(93.7 \%)$ completed the Participation Questionnaire. The statement that received the highest rating for why families enrolled in the study was they thought the study was researching an important community issue. The statements that received the highest ratings for why families continued to participate in the study was the family being kept well informed about the study's progress and that the study was well run. Conclusions-The low numbers of withdrawals and the high level of participation throughout the study suggests the strategies of (a) having a non-aggressive recruitment method, (b) maintaining regular contact with the participants and (c) ensuring participants were kept well informed of the study's progress and constantly encouraged to continue participation were successful. The results also suggest people involve themselves in research because they perceive it to be of value to the community, not simply for personal gain. They indicated that they maintained their participation because it was a well run study and they were kept well informed throughout the study. (F Epidemiol Community Health 2001;55:348-351)

Dr Hellard, Department of Epidemiology and Preventive Medicine, Monash Medical School, Alfred Hospital,

Commercial Road, Prahran,

3181, Australia

margaret.hellard@

med.monash.edu.au)

Accepted for publication

18 January 2001 research question. Compliance to follow up is critical for statistical validity.

The Water Quality Study was a double blinded randomised controlled trial conducted in Melbourne, Australia for 68 weeks between September 1997 and February 1999 investigating the gastrointestinal health effects of drinking water. The rate of gastroenteritis was measured in 600 families (2811 individuals) who were randomly allocated to have a real treatment unit (WTU) that removed microorganisms from the water or a sham WTU that did not alter the water. The WTU was installed in the participants' kitchens and was used as the families' main supply of drinking water.

A high level of commitment was required of the families. Participants recorded episodes of gastroenteritis in a Health Diary each week of the 68 weeks of the study. They answered multiple questionnaires regarding demographics, water and food consumption, food handling and storage, whether they thought their WTU was real or sham, and why the family enrolled in the study and continued to participate in the study, and provided faecal and blood samples.

Previous studies have suggested strict protocols for explaining the study and ongoing personal contact with the study team are important in maintaining participation. ${ }^{1-4}$ This contact needs to be organised in a flexible manner that suits study participants. ${ }^{1}$ Regular newsletters and similar activities were also reported to be beneficial in maintaining a high level of participation. ${ }^{45}$

Studies have reported that financial inducements increase the participants' response rates to questionnaires compared with participants who received no payment ${ }^{6}$; other studies reported participants found personal contact with the study staff more important than financial inducements. ${ }^{4}$

This paper describes the strategies used to maintain high level participation in a lengthy research study to ensure the study was adequately powered to provide an accurate and valid result. It reports the reasons given by participants as to why they enrolled and continued to participate in the study.

\section{Methods}

Three strategies were adopted to maintain high retention and participation rates throughout the 18 months of the study. The first strategy was to have a non-aggressive recruitment method, the second was to keep regular contact with the participants and the third was to ensure participants were kept well informed of the study's progress and to constantly encourage their ongoing participation. 
Table 1 Recruitment and screening data

\begin{tabular}{llll}
\hline & $\begin{array}{l}\text { First } \\
\text { screening }\end{array}$ & $\begin{array}{l}\text { Second } \\
\text { screeningt }\end{array}$ & Enrolment visit \\
\hline Total & 1488 & 1028 & $622 \ddagger$ \\
Eligible & 1028 & 647 & $615 \S$ \\
Ineligible & 459 & 358 & 7 \\
Other & 1 & 23 & 0 \\
\hline
\end{tabular}

^First screening-participants who telephone the study centre after receiving an invitation letter and/or pamphlet were asked questions regarding their eligibility and the family's eligibility to participate in the study. †Second screening - after participants had one to two weeks to read the information booklet they were telephone by study staff and asked if they were interested in participating in the study. Families who were interested in participating were asked a further screening questionnaire (similar to the first) to ensure they were eligible to participate in the study. ‡Only 622 families had enrolment visit (of which 600 participated in the study). The remaining 25 eligible families were not enrolled into the study because the study recruitment was complete. §Seven families were found to be ineligible at the enrolment visit (unable to install WTU in the kitchen). Fifteen families who were enrolled withdrew before WTU was installed and the study started. ( 3 because of the plumber being unable to install the WTU and 12 because of a change of mind). Otherincomplete or no contact for second screening.

Non-aggressive recruitment methods were developed to ensure families were not pressured to enrol and were clearly informed of the study before enrolment. Invitations to participate in the study were mailed to adults living in the study area and were distributed through primary schools, childcare centres, maternal health centres, and shopping malls and through advertisements in local newspapers. There were no direct telephone calls to participants' homes. Interested families were invited to telephone the study centre if they fulfilled the inclusion criteria outline in the letter. The study was fully explained when participants first telephoned the study centre, in a detailed plain language statement and when families were enrolled in the study.

The second strategy to maintain high retention and participation rates was to have regular contact with participants, with close monitoring of the return of Health Diaries and questionnaires. A research nurse was responsible for 150 families. The nurse visited the family three times during the study; telephoned families fortnightly and monitored the return rate of questionnaires via computer. The nurses recorded convenient times to telephone the families and had a back up telephone number of a contact person not participating in the study.

The third strategy to maintain participation was to inform participants of the study's progress via a monthly newsletter and to maintain the entire family's interest by having regular activities for the children, such as school holiday activity books and colouring competitions.

Participants were not paid to enter the study. They were reimbursed for extra electricity and water used during the study and could keep their WTU on completion of the study (sham WTUs were converted to a functioning WTU). Participants were sent a $\$ 1$ scratch lottery ticket and entered a lucky draw for a shopping voucher when they returned a completed Health Diary or collected a faecal specimen.

At the completion of the study families were asked why they enrolled and continued to participate in the Water Quality Study. The answers were weighted from 1 for strongly disagreed to 5 for strongly agreed using a five point Likert Scale.

\section{Results}

Altogether 74770 invitation letters were posted to people aged between 18 and 49 . A total of 25300 invitation pamphlets were delivered to the local primary schools, creches and kindergartens. There were 62029 households in the study area, of which 10918 fitted the study's selection criteria. ${ }^{7}$ The study recruited $5.49 \%$ of the available families.

One thousand four hundred and eighty eight people contacted the study centre after receiving an invitation letter and/or pamphlet; 1028 were eligible to participate in the study. Six hundred and fifteen families were enrolled in the study (table 1). Fifteen of these families did not participate and withdrew before the installation of the WTU.

Ninety three per cent of enrolled families completed the study. Forty one families withdrew, the most common reason was people sold their home and moved from the study area $(n=29)$. Families withdrew because of divorce or family crisis $(n=5)$, serious illness $(n=1)$ or renovations $(n=1)$. Four families were withdrawn for not completing their Health Diaries and one family withdrew because they did not like the taste of their water.

The completion rate of Health Diaries averaged $90.7 \%$ over the 68 weeks of data collection. The majority of adults in the study (more than $80 \%$ ) provided blood specimens at the beginning, middle and end of the study. One thousand and ninety one baseline stool specimens were submitted and 806 gastroenteritis faecal specimens were submitted. Of the 559 families who completed the study, 524 (93.7\%) replied to the Blinding Questionnaire and $523(93.5 \%)$ answered the Food Handling

Table 2 Why families agreed to participate in the Water Quality Study

\begin{tabular}{|c|c|c|c|c|}
\hline \multirow[b]{2}{*}{ We agreed to participate in the Water Quality Study because } & \multirow[b]{2}{*}{ Likert Score Median* } & \multicolumn{3}{|l|}{ Percentage } \\
\hline & & Disagree & Unsure & Agree \\
\hline We thought the study was researching an important issue for the community. & 4 & 0.4 & 2.9 & 97.0 \\
\hline It gave us an opportunity to do voluntary work for the community. & 4 & 8.3 & 9.2 & 81.8 \\
\hline We were interested in being involved in a scientific research study to see how they worked. & 4 & 12.6 & 12.0 & 75.0 \\
\hline We thought it was good for our children to be involved in a scientific research study. & 4 & 15.4 & 16.6 & 67.2 \\
\hline We were concerned about the quality of our drinking water. & 4 & 23.9 & 13.5 & 62.2 \\
\hline We wanted to get a water filter at the end of the study. & 3 & 47.9 & 14.7 & 37.1 \\
\hline Our children were having a lot of gastroenteritis and we though this would help find the cause. & 2 & 79.2 & 8.6 & 11.2 \\
\hline
\end{tabular}

${ }^{\star}$ Likert scale 1, strongly disagree; 2, disagree; 3, don’t know; 4, agree; 5, strongly agree. +Where percentages do not add up to $100 \%$ the data were missing. 
and Food Storage questionnaires. Over $80 \%$ of households completed at least one water consumption questionnaire. There were 1308 Gastroenteritis Questionnaires and 1838 Control Gastroenteritis Questionnaires answered.

Of the 559 families who completed the study, 524 (93.7\%) answered the Participation Questionnaire. The statement that received the highest rating (strongly agreed) for why families enrolled in the study was families thought the study was researching an important community issue (table 2). The statement that received the second highest rating was it gave the family an opportunity to perform voluntary work. The statements that received the highest ratings (strongly agreed) for why families continued to participate in the study were the family was kept well informed, the study was well run and the questionnaires were easy to complete (table 3). Families did not rate highly the chance to win money or be given a lottery scratch ticket.

\section{Discussion}

The combined strategies of explaining the study clearly, maintaining close contact and keeping participants interested and well informed were successful, resulting in a low withdrawal rate and high level of compliance in answering questionnaires and providing specimens. It is however not possible to identify which specific factors in the study were most important in recruiting and retaining participants in the study.

Participants rated regular and close contact with the research nurses, and being kept informed about the study's progress via the newsletter as important reasons for continuing to participate in the study. This is consistent with previous researchers who have variously reported ongoing personal contact and newsletters to be important in retaining participants. ${ }^{3-5}$

Small inducements were offered throughout the study to help maintain participants' enthusiasm and families were allowed to keep their WTU at the end of the study. Interestingly, participants did not report these as important factors in their continuing participation, citing performing voluntary work and participating in an interesting and well run study as more important. The relative importance of financial
KEY POINTS

- Clinical trials are expensive and difficult studies. A critical component to their success is maintaining a high level of participation.

- Compliance to follow up is critical for statistical validity.

- Non-aggressive recruitment methods may be important in maintaining long term participation in a clinical trial.

- Participants rated researching an important community issue and performing voluntary work were important reasons why they participated in research.

- Participants rated being kept well informed and the study being well run as important reasons they continued in a study.

inducements in maintaining ongoing participation in studies is uncertain. A study investigating methods of ensuring high follow up rates in participants with psychiatric and substance misuse disorders reported personal contact with the study staff was more important than financial inducements. ${ }^{4}$ A study investigating nutritional exposure before and during pregnancy reported participants valued a $\$ 100$ payment but did not offer evidence to support this statement. ${ }^{5}$ A study assessing the effect of direct payment or a lottery on the response rate to a mailed questionnaire found participants who were paid $£ 5$ were more likely to respond to the questionnaire compared with participants who received no payment or a lottery ticket. $^{6}$

An explanation for these different findings is financial inducements may be beneficial in a short-term study but other methods may be necessary to maintain interest and a high level of participation in a long term studies. An alternative explanation is people may not be totally candid in stating their motivation for participating in the study, preferring to state they participated for altruistic rather than pecuniary reasons. It is also important to note that participants in the Water Quality Study were a highly selected group who may have a strong inclination to participate in voluntary

Table 3 Why families continued to participate in the Water Quality Study

\begin{tabular}{|c|c|c|c|c|}
\hline \multirow[b]{2}{*}{ We continued to participate in the Water Quality Study because } & \multirow[b]{2}{*}{ Likert Score ${ }^{\star}$ Median } & \multicolumn{3}{|c|}{ Percentage } \\
\hline & & Disagree & Unsure & Agree \\
\hline The research nurses and the monthly newsletter kept us well informed. & 4 & 0.4 & 1.3 & 97.7 \\
\hline The study was well run. & 4 & 0.2 & 2.3 & 96.9 \\
\hline The questionnaires were simple to complete. & 4 & 2.3 & 1.7 & 95.2 \\
\hline The study did not take too much time. & 4 & 2.9 & 3.8 & 93.0 \\
\hline We found the study interesting. & 4 & 3.4 & 7.6 & 88.3 \\
\hline We did not want to let our research nurse down by not completing the questionnaires & 4 & 9.9 & 5.7 & 83.6 \\
\hline We promised to do the study and we do not like to break promises. & 4 & 9.9 & 5.7 & 83.6 \\
\hline It gave us an opportunity to do voluntary work for the community. & 4 & 9.3 & 9.2 & 80.2 \\
\hline The regular contact with my research nurse encouraged me to continue. & 4 & 8.6 & 11.3 & 79.2 \\
\hline $\begin{array}{l}\text { The children enjoyed being in the study because of the water bottles, colouring competitions and } \\
\text { holiday activities. }\end{array}$ & 4 & 22.5 & 24.4 & 52.3 \\
\hline We wanted to get a water filter at the end of the study. & 3 & 41.2 & 14.3 & 43.4 \\
\hline There was a chance of winning money from the scratch lottery ticket. & 2 & 56.8 & 12.6 & 29.6 \\
\hline There was a chance of winning a lucky draw of $\$ 100$. & 2 & 57.6 & 14.5 & 27.0 \\
\hline
\end{tabular}

*Likert scale 1, strongly disagree; 2 , disagree; 3, don't know; 4, agree; 5 , strongly agree. +Where percentages do not add up to $100 \%$ the data were missing. 
work. The factors motivating people to participate in research studies may vary between populations.

In conclusion, the high level of participation throughout the 68 weeks the Water Quality Study suggests the multiple strategies we used successful, although it is not possible to assess which strategy was the most important.

Information on what motivates people to maintain participation in research is limited. Our study results suggest people maintained their participation because the study was interesting and well run. The survey also suggested people involve themselves in research because they perceive it to be of value to the community, not simply for personal gain.

Funding: Cooperative Research Centre for Water Quality and Treatment, Water Services Association of Australia, Human Services Victoria, Melbourne Water, South East Water, Yarra Valley Water, City West Water.
Conflicts of interest: none.

1 Senturia YD, Mortimer KM, Baker D, et al. Successful techniques for retention of study participants in an inner-city population. Control Clin Trials 1998;19:544-54

2 Morse EV, Simon PM, Besch CL, et al. Issues of recruitment, retention, and compliance in communitybased clinical trials with traditionally undeserved populations. Appl Nurs Res 1995;8:8-14.

3 Dudley J, Jin S, Hoover D, et al. The Multicenter AIDS Cohort Study: retention after 9 1/2 years. Am $\mathcal{F}$ Epidemiol 1995;142:323-30.

4 BootsMiller BJ, Ribisl KM, Mowbray CT, et al. Methods of ensuring high follow-up rates: lessons from a longitudinal study of dual diagnosed participants. Subst Use Misuse 1998;33:2665-85.

5 Brown JE, Jacobs DR, Barosso GM, et al. Recruitment, retention and characteristics of women in prospective study of preconceptional risks to reproductive outcomes: experience of the Diana Project. Paediatr Pernat Epidemiol 1997;11:345-58

6 Roberts P-J, Roberts C, Sibbald B, et al. The effect of s direct payment or a lottery on questionnaire response rates: a randomised controlled trial. $\mathcal{F}$ Epidemiol Community a randomised contro $2000 ; 54: 71-2$.

7 Castles I. Census of Population and Housing 6 August 1991. Australia in profile. Canberra: Australian Bureau of Statistics, 1993: report no 2821.0. 